\title{
Recherches sur le cycle biologique de Pseudoleucocbloridium soricis (Soltys, 1952) dans les Pyrénées
}

\author{
par J. JOURDANE \\ Département de Biologie animale, Centre Universitaire, \\ avenue de Villeneuve, F 66025 Perpignan Cedex
}

\section{Résumé.}

Pseudoleucochloridium soricis évolue au stade sporocyste dans la glande digestive du Pulmoné terrestre Cepaea hortensis. La cercaire, du type microcerque, est émise dans le milieu extérieur et pénètre activement chez un deuxième Pulmoné terrestre où elle se développe en métacercaire libre dans la cavité péricardique.

Au plan de l'écologie, le cycle de $P$. soricis offre dans les Pyrénées deux caractères qui retiennent plus particulièrement l'attention:

- la transmission du parasite au deuxième hôte, bien que possible expérimentalement avec le même succès chez deux espèces d'Hélicidés ( $C$. hortensis et Euomphalia strigella), se réalise en fait dans la nature préférentiellement chez $E$. strigella. Le comportement trophique différent des deux Mollusques est interprété comme le facteur responsable de cette sélection;

- la contamination des musaraignes apparaît extrêmement faible (pourcentages jamàis supérieur à $1 \%$ ) dans toutes les stations, caractère qui contraste avec les valeurs toujours très fortes du niveau d'infestation du deuxième hôte (en moyenne $50 \%$ ). Cette particularité épidémiologique plaide en faveur de l'évolution probable de $P$. soricis chez un hôte définitif (Oiseau vraisemblablement) non encore découvert à ce jour.

\section{Summary.}

Findings on the life cycle of Pseudoleucochloridium soricis (Soltys, 1952) in the Pyrenees.

Pseudoleucochloridium soricis develops to the sporocyst stage in the digestive gland of the terrestrial Pulmonate Cepaea hortensis. The cercaria, microcercous in type, is liberated 
and actively penetrates a second terrestrial pulmonate where development to the free metacercarial stage takes place in the pericardial cavity.

Ecologically, the cycle of $P$. soricis in the Pyrenees, exhibites two characteristics which require particular attention:

- the transmission of the parasite to the second host, which, although experimentally possible, and with equal success, in two species of Helicide (C. hortensis and Euomphalia strigella) occurs in the wild preferentially in E. strigella. The differing trophic behaviour of the two molluscs is thought to be the factor responsible for this selection;

- the level of infection in shrews appears to be extremely low (never more than $1 \%$ ) in all sampling areas, a character which contraste with the persistantly high level of infection in the second host (at least $50 \%$ ). This epidemiological character points to the probable evolution of $P$. soricis in a definitive host (almost certainly a Bird) which has not yet been discovered.

L'espèce Pseudoleucochloridium soricis a été trouvée dans le tube digestif de trois genres de Micromammifères Insectivores de la zone paléarctique (Neomys, Sorex et Crocidura). Ce Digène est connu de Pologne (Soltys, 1952 et Pojmanska, 1959 et 1961), d'Allemagne (Stammer, 1955), de Bulgarie (Genov, 1966), de Russie (Shaldybin, 1953), de France (Jourdane, 1971), et de Roumanie (Chiriac et Popescu, 1973).

Malgré sa fréquence en Europe, ce parasite a suscité très peu de travaux sur le plan biologique et son cycle évolutif demeurait inconnu jusqu'ici. Les seules données sur sa biologie (Pojmanska, 1959 et 1961) sont relatives à la découverte du stade métacercaire dans le rein de plusieurs Pulmonés terrestres en Pologne.

Dans les Pyrénées, si l'adulte de $P$. soricis est extrêmement rare chez les musaraignes, la métacercaire est par contre très fréquente dans certaines stations, ce qui nous a conduit à rechercher à la fois expérimentalement et dans la nature les modalités du cycle de ce parasite.

\section{Recherches expérimentales}

\section{A. - Schéma du cycle.}

D'après nos recherches, le cycle de Pseudoleucochloridium soricis se déroule dans les Pyrénées selon le schéma suivant:

- l'œuf est éliminé avec les fèces dans le milieu extérieur où il sera ingéré par le Mollusque Helicidé Cepaea hortensis (Müller, 1774);

- le miracidium, libéré dans le tube digestif du Mollusque, évolue chez celui-ci en sporocyste I qui engendre à son tour des sporocystes. II se développant dans la glande digestive ; 
- les cercaires, produites par les sporocystes II, quittent le Mollusque premier hôte intermédiaire et pénètrent activement chez un deuxième Mollusque Hélicidé où elles évoluent en métacercaires non enkystées dans la cavité péricardique ; caires.

- l'hôte définitif s'infeste en ingérant les Mollusques parasités par les métacer-

\section{B. - Stades larvaires.}

\section{$1^{\circ}$ Sporocyste.}

La dissection de Cepaea hortensis trouvés naturellement infestés révèle que la glande digestive du Mollusque est totalement envahie par des sporocystes II localisés entre les acini. Ceux-ci, très nombreux, offrent deux aspects selon leur état de maturité :

- les sporocystes jeunes ont la forme de diverticules grêles, de $100 \mu$ de diamètre en moyenne, qui se détachent d'une poche centrale renfermant des balles germinales bien individualisées. Isolés de la glande digestive et placés dans une solution de Ringer, ils se montrent très mobiles : ils se contractent, s'allongent et se recourbent très activement. Sur des coupes colorées selon la technique de Mac Manus, les diverticules apparaissent limités par une assise de cellules germinatives contenant des inclusions PAS positives, vraisemblablement de nature glycogénique. Les cellules de la glande digestive du Mollusque sont bourrées de granulations semblables. A un stade plus avancé, les éléments germinatifs issus de la paroi des diverticules vont former progressivement des amas cellulaires dans la lumière des filaments ;

- les sporocystes mûrs (fig. $1 C$ ) sont constitués par des diverticules très ramifiés et renflés $(170 \mu$ de diamètre). Assez souvent, l'extrémité des diverticules se détache par constriction du reste de la masse sporocystique et évolue isolément. Toutes les branches du sporocyste, ainsi que les diverticules isolés, renferment des cercaires en voie de développement ayant pris naissance à partir des balles germinales centrales. A ce stade, les sporocystes n'ont plus de paroi cellulaire embryogène. La glande digestive du Mollusque, complètement envahie par le parasite, n'est plus représentée que par quelques îlots glandulaires, très involués, et dépourvus de granulations polysaccharidiques : le matériel PAS positif se trouve essentiellement localisé dans les sporocystes au niveau des cercaires. Sur le plan métabolique, la pathologie du Mollusque est donc marquée par un épuisement progressif des réserves glycogéniques.

\section{$2^{\circ}$ Cercaire.}

- Morphologie générale:

La différenciation des cercaires dans les sporocystes est très longue, de l'ordre de 3 à 4 mois selon nos estimations.

La cercaire appartient au groupe des cercaires microcerques (fig. $1 \mathrm{~A}$ ). Le corps de la cercaire est de forme lancéolée. Son tégument est glabre sur toute sa surface.

La queue est réduite à un moignon caudal à peine séparé du corps par un léger étranglement. 

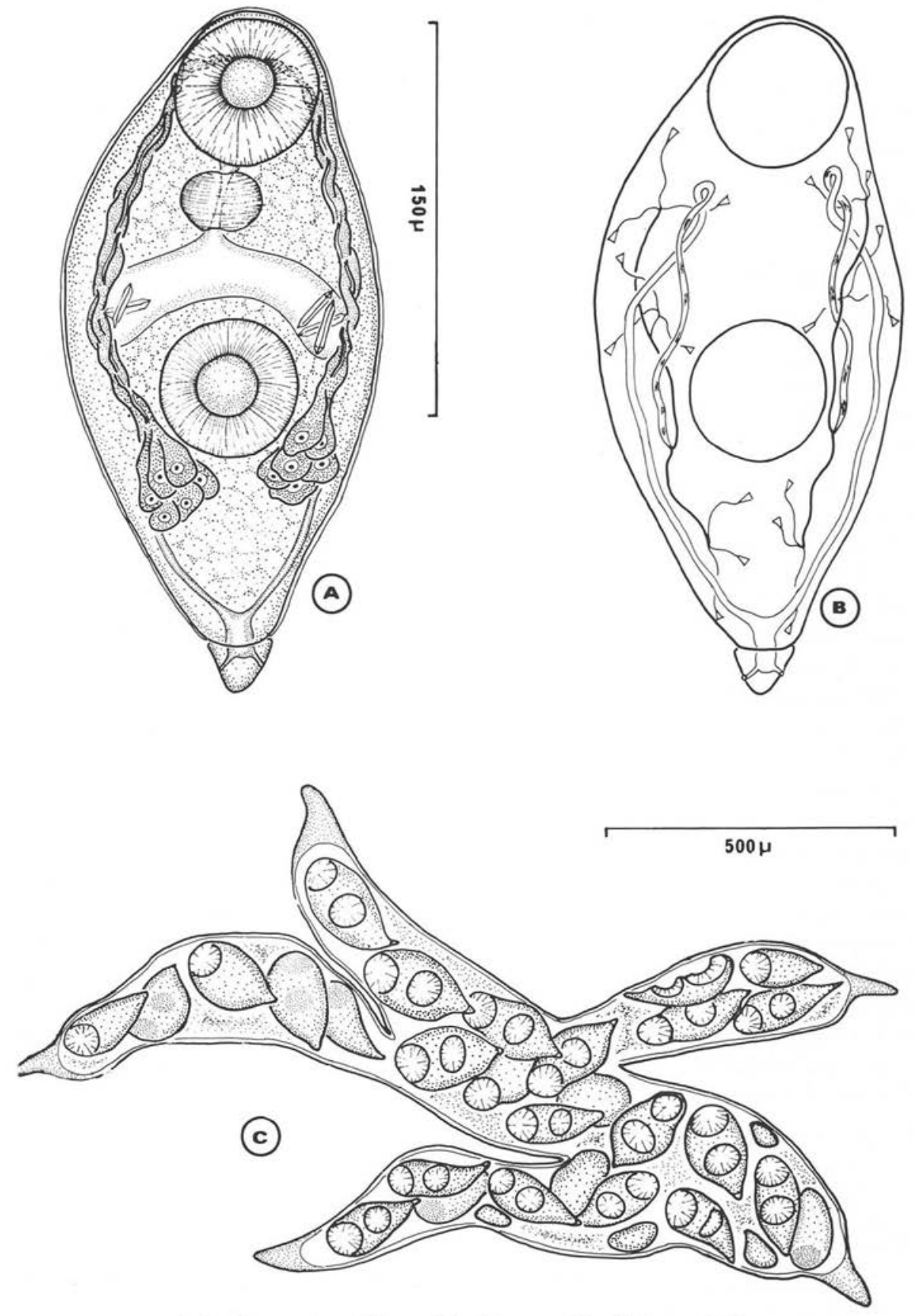

FIG. 1. - Pseudoleucochloridium soricis (Soltys, 1952).

A : Cercaire, morphologie générale. B : Cercaire, organisation du système excréteur. $\mathrm{C}$ : Sporocyste fils avec cercaires en voie de différenciation. 
Les deux ventouses, très développées, de forme circulaire, sont égales : l'acétabulum est situé légèrement en arrière du milieu du corps.

Les dimensions moyennes de la cercaire, mesurées sur le vivant, sont :

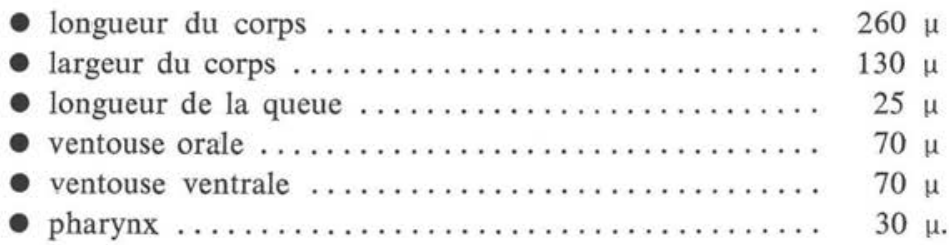

\section{- Organisation interne:}

Le système digestif est représenté par un phayrnx sphérique, volumineux, et deux. caecums courts se terminant au niveau du milieu de l'acétabulum. L'extrémité des caecums renferme des baguettes de nature cristalline, réfringentes en contraste de phase, dont nous n'avons pu préciser la composition.

Les glandes de pénétration comprennent 6 cellules par hémicorps, groupées au voisinage des bords postéro-latéraux de l'acétabulum. Leurs canaux longent de chaque côté les parois du corps et débouchent dans la région antérieure de l'ouverture orale.

Le système excréteur est du type sténostome (fig. $1 \mathrm{~B}$ ). On compte 18 cellules flammes se distribuant suivant la formule :

$$
2[(3+3)+(3)]
$$

Les cellules flammes sont drainées de chaque côté par un canal collecteur ascendant, dans la lumière duquel battent des pinceaux de cils. Chaque canal ascendant se recourbe au niveau du pharynx et se continue par un tronc collecteur qui suit les côtés de la cercaire jusqu'à la vessie. Celle-ci, peu différenciée, s'étend dans la queue et se déverse à l'extérieur par deux petits conduits divergents qui débouchent sur les côtés latéraux du moignon caudal.

\section{- Comportement :}

Dans les conditions expérimentales, l'émission des cercaires est obtenue en plaçant les Mollusques infestés dans de petits cristallisoirs au fond recouvert de papier filtre humide, de manière à réaliser un milieu saturé d'humidité. Les cercaires émises dans le mince film d'eau du papier filtre sont animées de mouvements de contraction répétés : on peut souvent les observer fixées par leur acétabulum, relevant et abaissant de manière synchrone et rythmique leurs extrémités antérieures et postérieures. Leurs déplacements dans l'espace, effectués uniquement par reptation, sont très limités.

Après rencontre avec le deuxième hôte intermédiaire, la cercaire gagne par reptation l'orifice urinaire, s'y introduit et remonte le conduit rénal jusqu'à la cavité péricardique. La progression de la larve sur les téguments du Mollusque en direction de l'orifice urinaire, le plus souvent localisé très rapidement, nous semble être guidée par un authentique chimiotropisme. 


\section{$3^{\circ}$ MÉtACERCAIre.}

La réalisation expérimentale du cycle de $P$. soricis nous a permis de suivre la différenciation progressive de la métacercaire chez le deuxième hôte intermédiaire. La métacercaire, jamais enkystée, se développe dans la cavité péricardique d'un Mollusque Hélicidé.
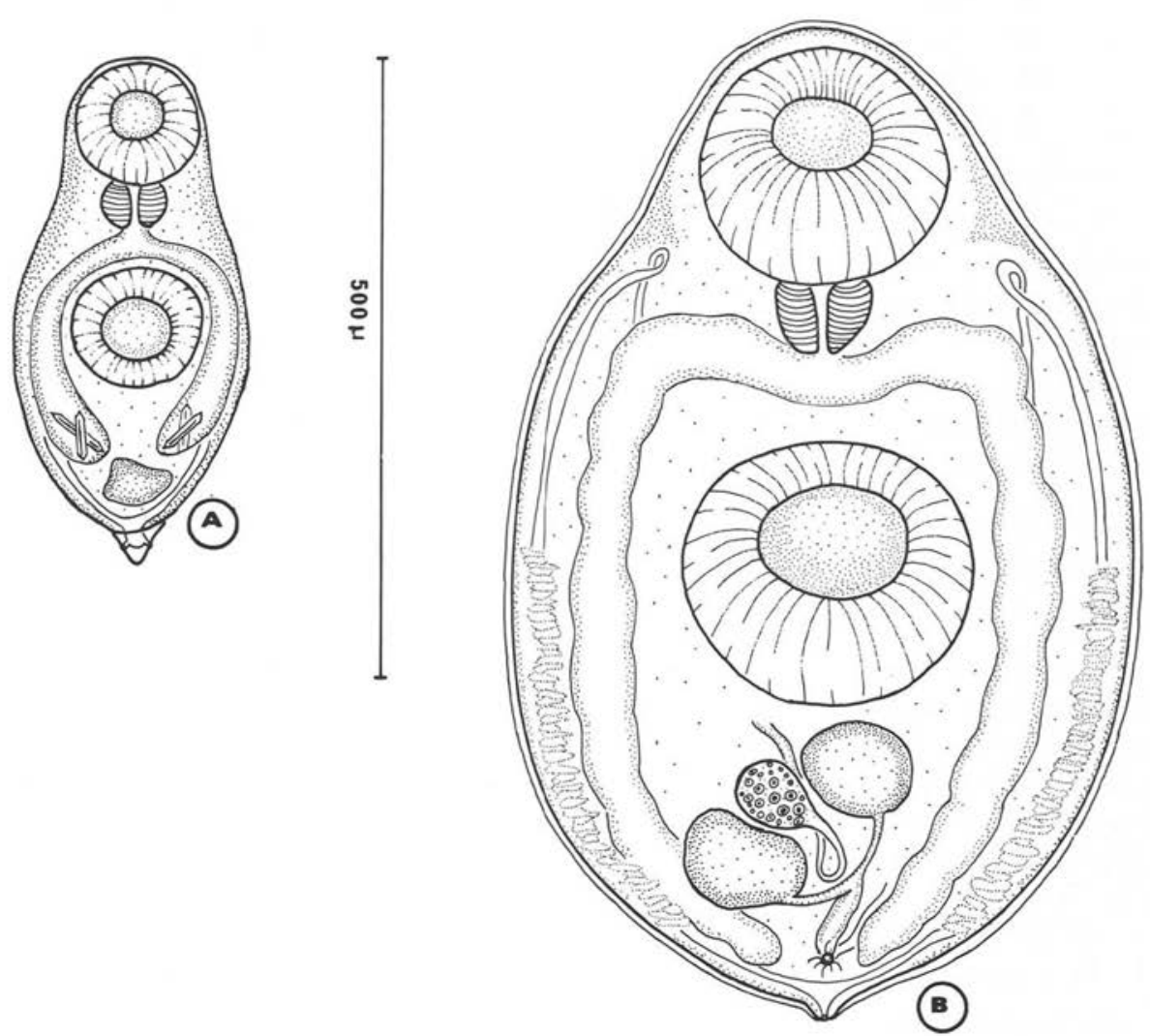

Fig. 2. - Pseudoleucochloridium soricis (Soltys, 1952).

A : Métacercaire âgée de 30 jours. B : Métacercaire infestante.

La morphogénèse de la métacercaire, même à la température du laboratoire $\left(+20^{\circ} \mathrm{C}\right)$, est très lente.

La métacercaire de 30 jours (fig. 2 A) mesure environ $400 \mu$ de longueur. Sur le plan anatomique, elle se différencie de la cercaire par la longueur de ses caecums qui dépassent nettement le niveau du bord postérieur de l'acétabulum, par la disparition totale des glandes de pénétration et de leurs canaux et par la différenciation des cellules germi- 
natives. Deux caractères de la cercaire persistent encore chez ces jeunes métacercaires: l'appendice caudal et les formations cristallines à l'intérieur des caecums.

L'évolution ultérieure de la métacercaire va être marquée à la fois par une augmentation considérable de taille et par la morphogenèse de l'appareil génital de l'adulte. D'après nos observations, nous pensons que la morphogenèse de la métacercaire dure de cinq à six mois.

La métacercaire infestante (fig. $2 \mathrm{~B}$ ) présente un corps très renflé au niveau de l'acétabulum. Son tégument, épais, est dépourvu de toute spinulation. L'appendice caudal a disparu chez la plupart des individus.

Les ventouses, circulaires, sont très développées par rapport au corps.

Le système digestif comprend un pharynx globuleux, accolé à la ventouse orale, de $165 \mu$ de diamètre environ, et deux caecums digestifs qui s'étendent jusqu'au niveau du pore génital.

Les glandes sexuelles, bien différenciées, occupent une position post-acétabulaire et offrent la disposition caractéristique des Brachylaemidae: ovaire situé entre les deux testicules qui sont alignés longitudinalement. Les testicules, ovoïdes, mesurent environ $130 \times 100 \mu$. L'ovaire, sphérique, a $24 \mu$ de diamètre en moyenne. Les glandes vitellogènes sont peu différenciées chez la métacercaire. Leur extension selon deux champs latéro-postérieurs développés entre le niveau du pore génital et celui du milieu de l'acétabulum peut toutefois être discernée. Le pore génital, ventral, s'ouvre à mi-distance entre le testicule postérieur et l'extrémité du ver.

Les mensurations de la métacercaire, relevées chez 20 larves vivantes, sont :

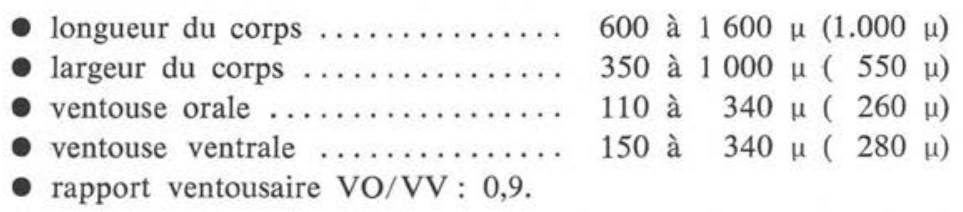

Il ressort de l'analyse de ces mensurations que la taille des métacercaires infestantes peut varier dans de très grandes proportions.

La présence de métacercaires dans la cavité péricardique s'accompagne de lésions histologiques importantes des tissus (fig. 3). Le cœur apparaît comme l'organe le plus atteint: sa paroi est considérablement amincie et ses cellules musculaires superficielles sont souvent disjointes. Les lésions sont la conséquence des actions mécanique (succion) et enzymatique du parasite. Les caecums de la métacercaire sont souvent remplis d'éléments celullaires pigmentés dont l'origine myocardique est indéniable.

\section{C. - Recherches expérimentales des hôtes.}

$1^{\circ}$ Détermination du SPECTRE DEs deuxièmes hôtes.

Nous avons tenté l'infestation avec la métacercaire de $P$. soricis des Pulmonés terrestres les plus fréquemment rencontrés dans nos biotopes d'étude :

Euomphalia strigella (Draparnaud, 1801). 
Cepaea hortensis (Müller, 1774).

Succinea putris (Linné, 1758).

Arion lusitanicus (Mabille, 1870).

Agriolimax reticulatus (Müller, 1774).

Seules les expériences réalisées avec $E$. strigella et $C$. hortensis ont été positives.

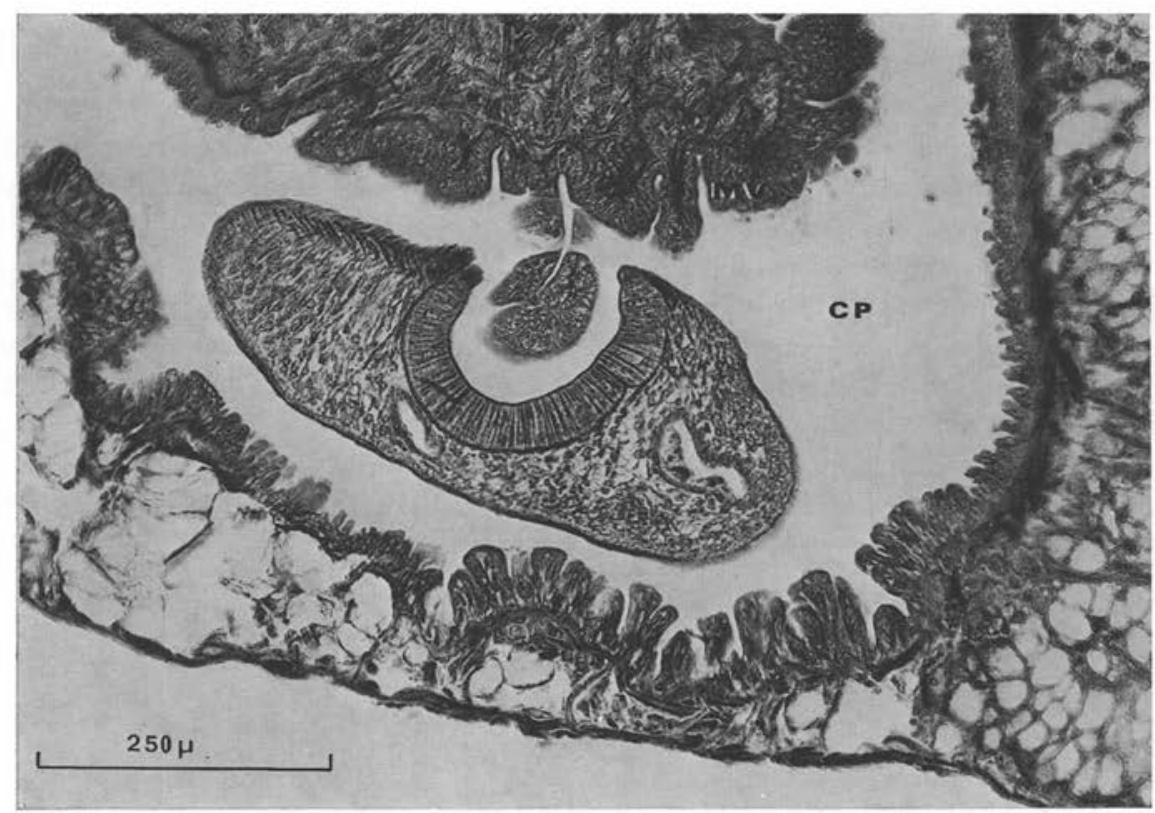

FIg. 3. - Pseudoleucochloridium soricis (Soltys, 1952).

Métacercaire dans la cavité péricardique (CP) du Mollusque deuxième hôte intermédiaire. Noter les dégradations importantes du myocarde ventriculaire (MV) consécutives au parasitisme.

Sur le plan de la dynamique de la transmission du parasite au deuxième hôte, il nous paraît important de souligner que celle-ci ne se réalise que si l'hôte vient en contact avec la cercaire qui elle, nous l'avons précisé, ne peut effectuer que des déplacements très limités sur le substrat.

Il nous a paru intéressant de rechercher expérimentalement si les deux hôtes possibles $E$. strigella et $C$. hortensis avaient la même «valeur» pour la cercaire de $P$. soricis. A cet effet, nous avons réalisé une série d'infestations dans lesquelles les deux Pulmonés étaient mis en « compétition » dans un récipient renfermant un lot de cercaires.

Dans tous les cas, les résultats obtenus n'ont pas révélé de différence quantitative dans la contamination des deux Hélicidés. 
$2^{\circ}$ INFESTATION DE L'HôTE DÉFINITIF.

Nous avons réussi l'infestation avec les métacercaires de $P$. soricis des Soricidés Neomys fodiens et Sorex araneus.

L'analyse de l'évolution de la parasitose expérimentale révèle que celle-ci ne se maintient pas chez les musaraignes au-delà de 48 heures. Pendant cette durée relativement courte, le parasite parvient néanmoins à maturité et se reproduit chez son hôte : l'autopsie de musaraignes, 36 heures et 24 heures après l'infestation expérimentale, livre des Digènes mûrs et ovigères pour la plupart.

\section{Modalités du cycle dans la nature}

Le développement de Pseudoleucochloridium soricis s'effectue dans l'Est des Pyrénées chez les hôtes suivants :

Premier hôte intermédiaire .......... Cepaea hortensis.

Deuxième hôte intermédiaire .......... Cepaea hortensis. Euomphalia strigella.

Hôte définitif ................. Neomys fodiens. Sorex araneus. Sorex minutus.

Au niveau du Mollusque premier hôte, la fréquence de la parasitose est toujours très faible : elle oscille suivant les stations entre 1 et $2 \%$. Ces valeurs sont à rapprocher de celles signalées chez la plupart des cycles de Brachylaemidae: dans tous les cycles connus pour lesquels nous avons des données quantitatives sur le parasitisme du premier hôte, les taux d'infestation ne dépassent jamais en effet $5 \%$.

Au niveau du deuxième hôte, le taux de contamination est très différent pour les deux Pulmonés et varie beaucoup selon les stations : nous avons reporté sur le tableau I les valeurs observées dans deux stations distinctes sur le plan écologique : la station A est représentée par les berges d'une rivière, la station B est constituée par un bosquet d'Aunes.

TABleau I. - Données quantitatives sur la parasitose des deuxièmes hôtes intermédiaires dans deux stations pyrénéennes.

\begin{tabular}{|c|c|c|c|c|}
\hline & & $\mathrm{Nb}$. disséqués & $\mathrm{Nb}$. parasités & $\%$ parasités \\
\hline \multirow{2}{*}{ Station A $\ldots \ldots$. } & E. strigella & 172 & 44 & 25,5 \\
\hline & C. hortensis & 145 & 15 & 10,3 \\
\hline \multirow{2}{*}{ Station B ........ } & E. strigella & 28 & 27 & 96,4 \\
\hline & C. hortensis & 30 & 6 & 20 \\
\hline
\end{tabular}


Deux observations intéressantes ressortent de la lecture de ce tableau :

- d'une station à l'autre, le taux de parasitisme fluctue de façon très importante chez un même hôte : de 25 à $96 \%$ dans le cas de E. strigella, de 10 à $20 \%$ dans le cas de $C$. hortensis. Ces différences nous semblent essentiellement liées aux caractéristiques écologiques très dissemblables des deux biotopes. L'existence notamment d'une population de musaraignes très dense en $\mathrm{B}$ nous paraît représenter un facteur particulièrement favorable au déroulement du cycle dans ce foyer;

- au sein d'une même station, le niveau d'infestation métacercarienne des deux Pulmonés se révèle très différent. En $\mathrm{A}$, les pourcentages sont significativement différents à $1 \%$ près (écart réduit, $\varepsilon=2,6$ ) : en $\mathrm{B}$, la différence est significative à $10{ }^{7}$ près (écart réduit, $\varepsilon=5,9$ ). E . strigella doit être considéré comme l'hôte habituel de $P$. sori. cis dans notre région. Nous avons toutefois noté plus haut que les deux Mollusques mis en «compétition» en laboratoire avaient la même valeur pour la cercaire de $P$. soricis. L'éthologie des deux Hélicidés liée à leur alimentation peut à notre avis expliquer au moins en partie les différences de parasitisme observées dans la nature : E. strigella est une espèce essentiellement détritivore, $C$. hortensis se nourrit préférentiellement d'organes végétaux frais. Il en résulte que les deux Mollusques colonisent en période d'activité trophique des strates végétales différentes: $C$. hortensis évolue sur les organes végétatifs des plantes du biotope, E. strigella recherche sa nourriture à la surface du sol. Par ailleurs, nous pensons que les lésions pathologiques décelées chez les Cepaea infestés par les sporocystes de $P$. soricis contraignent ces individus, par l'épuisement qu'elles induisent, à demeurer aussi au niveau du tapis végétal. Dès lors, la dissémination des cercaires s'effectuerait en grande partie à la surface du sol, ce qui à l'évidence rendrait possible une contamination cercarienne des Euomphalia pendant toute leur phase trophique. L'infestation des Cepaea ne se réaliserait quant à elle qu'au tout début de leur période d'activité, avant leur ascension sur les végétaux.

Le processus de sélection de l'hôte habituel (E. strigella) parmi les hôtes effectifs du cycle relèverait donc pour beaucoup de facteurs trophiques.

Le niveau d'infestation des musaraignes par $P$. soricis s'est révélé extrêmement faible dans toutes les stations pyrénéennes prospectées: nous n'avons jamais recencé plus de $1 \%$ de musaraignes parasitées. Ce niveau de parasitisme est tout à fait superposable à ceux notés dans les autres stations européennes: Soltys donne, en Pologne, dans son enquête épidémiologique portant sur 3444 musaraignes, le chiffre de $0,9 \%$; Shaldybin indique, en Russie, 1,8\% ; Genov et Dimitrova mentionnent, en Bulgarie, $2 \%$. En Pologne, où nous avons des données sur le taux de parasitisme du deuxième hôte, et plus encore dans les Pyrénées, le déséquilibre très marqué entre la contamination du deuxième hôte et celle de l'hôte définitif ne peut manquer d'éveiller notre intérêt. Nous ne pouvons en l'état actuel de nos connaissances donner une explication définitive à cette particularité épidémiologique. L'hypothèse de travail que nous avons retenue consiste à admettre que ce Digène, bien qu'évoluant indiscutablement jusqu'à la maturité chez les musaraignes, parasite vraisemblablement d'autres hôtes (Oiseaux en particulier). La lon- 
gévité très brève du parasite (inférieure à 48 h) chez les musaraignes plaiderait en faveur de cette hypothèse.

\section{Conclusion}

L'espèce Pseudoleucochloridium soricis est traditionnellement rangée par la plupart des auteurs dans la famille des Leucochloridiidae sur la base de caractères morphologiques. D'après nos résultats, il apparaît cependant que les modalités du développement de $P$. soricis montrent beaucoup de différences par rapport à celles des Leucochloridiidae : chez ceux-ci notamment, l'évolution larvaire s'effectue en totalité chez le même hôte intermédiaire, la cercaire s'enkystant sur place à l'intérieur du sporocyste. Le cycle biologique de $P$. soricis s'inscrit par contre parfaitement dans le cadre des processus de transmission actuellement connus chez les Brachylaemidae sensu stricto. L'évolution de $P$. soricis s'apparente plus étroitement à celle de trois espèces de cette dernière famille :

- Dollfusinus frontalis, Biocco et Ferretti, 1958, parasite des sinus frontaux des hérissons et lérots circumméditerranéens ;

- Panopistus pricei Sinitzin, 1931, parasite intestinal de la Musaraigne américaine Blarina brevicauda; Amérique.

- Postharmostomum helicis (Cleidy, 1847), parasite intestinal de plusieurs Rongeurs en

La comparaison des quatre cycles permet de relever les parentés suivantes :

- l'évolution des parasites au stade larvaire se fait chez deux Mollusques Pulmonés de la même espèce ou d'espèces différentes ;

- les cercaires, du type microcerque, sont très voisines sur le plan anatomique ;

- le développement des métacercaires se réalise dans la cavité péricardique du Mollusque deuxième hôte intermédiaire.

Par les caractères de l'écologie de son cycle, l'espèce Pseudoleucochloridium soricis paraît donc devoir être classée préférentiellement parmi les Brachylaemidae, choix que n'interdit par ailleurs aucun caractère morphologique.

\section{Bibliographie}

Chiriac (H.) et Popescu (A.), 1973. - Recherches sur l'helminthofaune de quelques espèces d'Insectivores (Mammalia) en corrélation avec le régime alimentaire. St. si cerc. biol., $25,117-125$.

Genov (T.) et Dimitrova (E.), 1966. - On the helminthofauna of Insectivorous mammals in Bulgaria. Bull. cent. Helminth. Lab., 11, 125-159.

JouRdANE (J.), 1971. - Helminthes parasites des Micromammifères des Pyrénées-Orientales. II. Les Plathelminthes de Soricinae. Ann. Parasitol. hum. comp. 46, 553-574. 
PoJMAnSKa (T.), 1959. - Metacercariae of some Brachylaemidae (Trematoda) in Land snails of the Bialowieza National Park. Acta Parasit. polon., 7, 343-369.

POJMANSKA (T.), 1961. - Investigations on the occurence and biology of Trematodes of Sorex araneus L. in Bialowieza National Park. Acta Parasit. polon., 9, 305-330.

Shaldybin (L. G.), 1953. - New Trematodes from Insectivores. Contrib. helminthol., Moscou, 748-755.

Soltys (A.), 1952. - The helminths of common shrew (Sorex araneus L.) of the National Park of Bialowieza (Poland). Ann. U.M.C.S., 6, 165-209.

Stammer (H. J.), 1955. - Die parasiten deutschen Kleinsäuger. Verhandl. d. Deutsh. Zool. Gesell. in Erlangen, 362-390. 